\title{
Subpicosecond Laser Timing Stabilization
}

\author{
MARK J. W. RODWELL, DAVID M. BLOOM, FELLOW, IEEE, AND \\ KURT J. WEINGARTEN, MEMBER, IEEE
}

\begin{abstract}
The $0.25 \mathrm{~Hz}-25 \mathrm{kHz}$ pulse timing fluctuations of $\mathrm{Nd}$ : YAG CW-mode-locked laser are reduced from $20.6 \mathrm{ps}$ to less than $0.30 \mathrm{ps}$ rms by an electronic phase-lock loop. Stabilizer design considerations include the laser phase control characteristics, phase detector additive noise, and spurious detection of the laser amplitude noise by the loop phase detector. Applications include synchronization of multiple picosecond pulsed lasers, and synchronization of lasers and electronic signal sources in picosecond physical measurements.
\end{abstract}

\section{INTRODUCTION}

$\mathrm{M}$ ODE-LOCKED laser systems, in conjunction with optical pulse compressors, generate extremely short optical pulses, making these instruments attractive for a variety of time-resolved measurements, including electrooptic sampling [1]-[4], photo-enhanced electron-beam probing of integrated circuits [5], carrier dynamics and electromagnetic transient measurements in semiconductors [6], [7], optical waveform sampling [8], and timeresolved spectroscopy [9]. In these experiments, the system under test is frequency pumped (excited) by the pulsed laser and subsequently probed by a delayed portion of the same optical pulse, suppressing the effect of pulse timing fluctuations.

In many experiments, laser pulse excitation of the system under test is either inconvenient or is an unrepresentative experimental condition. In electrooptic sampling experiments [4], the integrated circuit under test is best excited by electronic signal generators. In other laser experiments, it may be most desirable to excite and probe the tested system with different lasers. In these cases, fluctuations of the laser timing degrade the time resolution in proportion to the uncertainty of the pulse arrival time, and introduce noise in proportion to the laser timing deviation times the time derivative of the experimental response.

Picosecond physical measurements can be performed with multiple mode-locked lasers and multiple electronic sources if all signal frequencies and pulse repetition rates

\footnotetext{
Manuscript received June 16, 1988; revised October 18, 1988. This work was supported by the U.S. Air Force Office of Scientific Research under Contract F49620-85-K-0016 and by the Wright-Patterson Air Force Base Avionics Laboratory under Contract F33615-86-C-1126.

M. J. W. Rodwell was with the Edward L. Ginzton Laboratory, Stanford University, Stanford, CA 94305 . He is now with the Department of Electrical and Computer Engineering, University of California, Santa Barbara, CA.

D. M. Bloom is with the Edward L. Ginzton Laboratory, Stanford University, Stanford, CA 94305.

K. J. Weingarten is with Lightwave Electronics, Inc., Mountain View, CA.

IEEE Log Number 8826284
}

are locked to a common frequency standard. Microwave frequency synthesizers use feedback systems to synchronize a high-frequency oscillator to a precision crystal oscillator. We have used similar techniques to synchronize a mode-locked laser to a stable radio-frequency synthesizer, thereby suppressing the laser timing fluctuations.

\section{Measurement of Timing Fluctuations by Spectral Techniques}

Fluctuations in mode-locked lasers include variations in both pulse intensity and pulse timing. These random processes, which are described by their power spectral densities, can be determined by measurement of the power spectrum of the laser intensity, as demonstrated by von der Linde and Kluge [10], [11]. Spectral techniques are widely used to determine and to specify the timing (phase) stability of radio-frequency and microwave signal sources [12]-[16]. First consider a single-frequency signal with sinusoidal amplitude and phase modulation:

$$
\begin{aligned}
v_{a m / p m}(t)= & \left(1+N_{c} \cos \omega_{m} t+N_{s} \sin \omega_{m} t\right) \\
& \times \exp \left(j\left(\omega_{l} t+\theta_{c} \cos \omega_{m} t+\theta_{s} \sin \omega_{m} t\right)\right) .
\end{aligned}
$$

If the modulations $N_{c}, N_{s}, \theta_{c}$, and $\theta_{s}$ are small, the Fourier transform $V_{a m / p m}(\omega)$ of $v_{a m / p m}(t)$ is

$$
\begin{aligned}
V_{a m / p m}(\omega)= & 2 \pi\left(\delta\left(\omega-\omega_{l}\right)+C_{u} \delta\left(\omega-\omega_{l}-\omega_{m}\right)\right. \\
& \left.+C_{l} \delta\left(\omega-\omega_{l}+\omega_{m}\right)\right)
\end{aligned}
$$

where

$$
\begin{array}{ll}
C_{u}=C_{a}+C_{p} & C_{l}=C_{a}^{*}-C_{p}^{*} \\
C_{a}=N_{c} / 2+j N_{s} / 2 & C_{p}=-\theta_{s} / 2+j \theta_{c} / 2 .
\end{array}
$$

The amplitude modulation and phase modulation of a signal are determined from the Fourier spectrum by decomposing the upper and lower sidebands $C_{u}$ and $C_{l}$ into phase and amplitude modulation sidebands $C_{p}$ and $C_{a}$ :

$$
C_{a}=\frac{C_{u}+C_{l}^{*}}{2} \quad C_{p}=\frac{C_{u}-C_{l}^{*}}{2} .
$$

The intensity of the pulse train from a mode-locked laser can be approximated as a series of Gaussian pulses with timing and amplitude fluctuations as given by

$$
\begin{aligned}
P(t)= & \bar{P} T(1+N(t)) \times \sum_{n=-\infty}^{n=+\infty} \frac{1}{\sqrt{2 \pi} \sigma_{t}} \\
& \cdot \exp \left(-\left(t-T_{0}-n T_{l}-J(t)\right)^{2} / 2 \sigma_{l}^{2}\right)
\end{aligned}
$$


where $P(t)$ is the laser intensity, $\bar{P}$ is the beam's average intensity, $T_{l}$ is the pulse repetition period, $\sigma_{t}$ is the rms pulse duration, $N(t)$ is the normalized pulse-intensity variation, $J(t)$ is the timing fluctuation of the pulse train, and $T_{0}$ is the static timing offset of the pulse train. The laser intensity then has a power spectral density $S_{P}(\omega)$ approximated to second order in $n \omega_{l} \sigma_{J}$ by

$$
\begin{aligned}
S_{P}(\omega) \simeq & \bar{P}^{2} e^{-\omega^{2} \sigma_{l}^{2}} \sum_{n=-\infty}^{n=+\infty}\left(\left(1-n^{2} \omega_{l}^{2} \sigma_{J}^{2}\right) 2 \pi \delta\left(\omega-n \omega_{l}\right)\right. \\
& +\left(1-n^{2} \omega_{l}^{2} \sigma_{J}^{2}\right) S_{N}\left(\omega-n \omega_{l}\right) \\
& \left.+n^{2} \omega_{l}^{2} S_{J}\left(\omega-n \omega_{l}\right)\right)
\end{aligned}
$$

where $\sigma_{J}$ is the standard deviation of $J(t)$ (i.e., the rms timing jitter), $\omega_{l}=2 \pi / T_{l}, S_{N}(\omega)$ is the power spectral density of $N(t)$, and $S_{J}(\omega)$ is the power spectral density of $J(t)$. In this text, two-sided power spectral densities are used, i.e., $S_{J}(\omega) \equiv \int_{-\infty}^{+\infty}\langle J(t) J(t+\tau)\rangle e^{-j \omega t} d \tau$ where $\langle\cdots\rangle$ denotes the mean value (statistical expectation). The spectrum of the laser intensity, measured using a photodiode and a microwave spectrum analyzer, is a series of lines at multiples of $\omega_{l}$ plus a series of amplitude noise sidebands $S_{N}\left(\omega-n \omega_{l}\right)$ and phase noise sidebands $n^{2} \omega_{l}^{2} S_{J}\left(\omega-n \omega_{l}\right)$. At harmonics of sufficiently low order (Fig. 1), the amplitude-noise sidebands predominate. The phase-noise sidebands have power proportional to $n^{2}$ and predominate for harmonics of high order (Fig. 2). The spectral density of the timing jitter is determined by the relative power of the laser harmonic and its associated sidebands. At very high harmonics, (6) is inaccurate because of higher order terms in $n \omega_{l} \sigma_{J}$ and because of spectral terms associated with pulse-width fluctuations [11]. Also defined in Fig. 2 is the single-sideband phase noise spectral density of the laser $n$th harmonic $L_{n}(\omega)=n^{2} \omega_{l}^{2} S_{J}(\omega)$, an NBS-defined parameter used to specify the phase stability of microwave oscillators. $L(f)$ $=L(\omega / 2 \pi)$ is the ratio of the power in one phase modulation sideband, in a $1 \mathrm{~Hz}$ integration bandwidth, to the total spectral power, at an offset $f \mathrm{~Hz}$ away from the oscillation frequency ("carrier") [12]. Following the terminology of the microwave literature, $L(\omega)$ and $S_{N}(\omega)$ are specified in decibels. $L(f=100 \mathrm{~Hz})=L(\omega=200 \pi$ $\left.\mathrm{s}^{-1}\right)=10^{-10} \mathrm{~s}$ is written as $L(f)=-100 \mathrm{dBc}(1 \mathrm{~Hz})$ at $f=100 \mathrm{~Hz}$.

Spectrum analyzers display the spectra of random signals as the noise power integrated over the analyzer resolution bandwidth. Analyzers employing filters and envelope detectors show small calibration errors for random signals, arising from the differences between half-power bandwidth and equivalent noise bandwidth, and between envelope detection and power detection [13]. Characteristics and methods of measurement of phase noise are discussed in several application notes from Hewlett-Packard [12]-[15] and by Robins [16].

With $S_{J}(\omega)$ determined, the root-mean-squared (rms)
Amplitude-Noise Sidebands: Low Harmonics

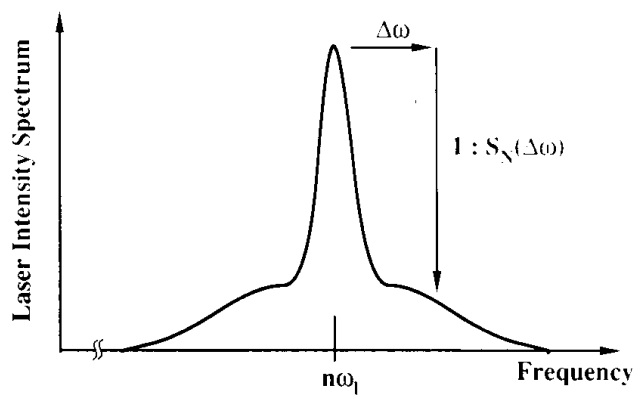

Fig. 1. Measurement of laser amplitude noise. Amplitude-modulation sidebands are observed at low harmonics of the laser pulse repetition frequency $\omega_{l}$.

Phase-Noise Sidebands: High Harmonics

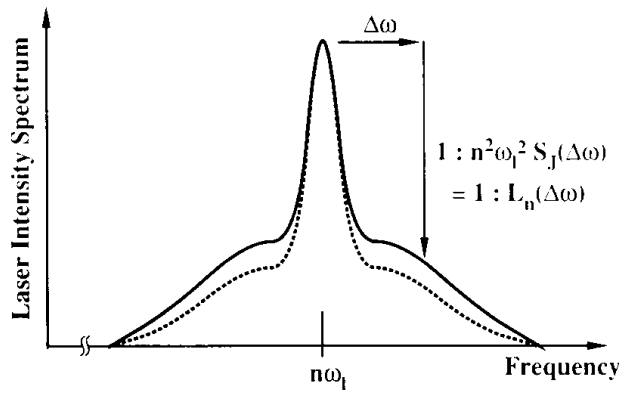

Fig. 2. Measurement of laser timing jitter. Phase-modulation sidebands $L_{n}(\Delta \omega)$ are observed at high harmonics of the laser pulse repetition frequency. Through integration of these sidebands, the rms laser timing jitter is determined.

jitter in the frequency range $\left(\omega_{\text {low }}, \omega_{\text {high }}\right)$ can be found:

$$
\sigma_{J} \equiv \sqrt{\left\langle J(t)^{2}\right\rangle}=\sqrt{\frac{1}{\pi} \int_{\omega_{\text {low }}}^{\omega_{\text {high }}} S_{J}(\omega) d \omega}
$$

If the jitter spectral density $S_{J}(\omega)$ has low-frequency components varying as $\sim 1 / \omega^{k}$, (7) may be large or unbounded given integration from $\omega_{\text {low }}=0 \mathrm{~s}^{-1}$. The change in laser timing over the duration $\Delta T$ of an experiment is given by a weighted integral of the timing jitter spectral density:

$$
\begin{aligned}
& \left\langle(J(t+\Delta T)-J(t))^{2}\right\rangle \\
& \quad=\frac{2}{\pi} \int_{0}^{+\infty} S_{J}(\omega)(1-\cos (\omega \Delta T)) d \omega .
\end{aligned}
$$

Because $(1-\cos (\omega \Delta T))$ varies as $(\omega \Delta T)^{2}$ for $(\omega \Delta T)$ $\ll 1$, jitter components with frequency below $\omega_{\text {low }}=$ $\pi / 2 \Delta T$ contribute little to changes in laser timing observed over the period $\Delta T$. Here, rms laser timing jitter will be specified over a stated frequency range ( $\omega_{\text {low }}$, $\omega_{\text {high }}$, as in (7). Integration from $f_{\text {low }}=0.25 \mathrm{~Hz}$ is appropriate for evaluation of timing variations occurring during an experiment of $1 \mathrm{~s}$ duration. 


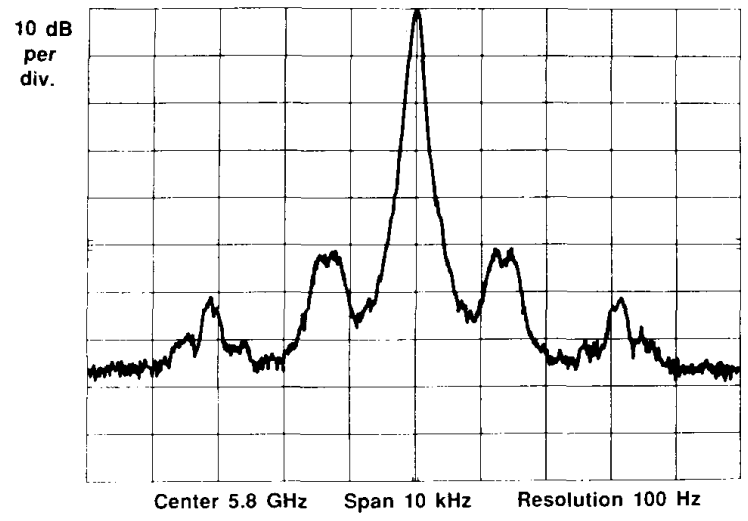

Fig. 3. Coherent Antares $1.06 \mu \mathrm{m} \mathrm{Nd}$ : YAG laser phase-noise sidebands, $500 \mathrm{~Hz}-5 \mathrm{kHz}$, measured on the 70 th harmonic of the $70 \mathrm{MHz}$ pulse repetition rate.

The phase noise of several Nd:YAG lasers has been measured, and jitter (at rates of $0.25 \mathrm{~Hz}$ and above) in the range of $1.8-20.6$ ps has been determined. Fig. 3 shows the measured phase noise sidebands in a $10 \mathrm{kHz}$ span about the 70th harmonic of the $70 \mathrm{MHz}$ pulse repetition rate of a Coherent Antares mode-locked Nd: YAG laser, measured with a microwave spectrum analyzer (HewlettPackard model HP8662A). The timing jitter of this laser is $1.8 \mathrm{ps} \mathrm{rms}(0.25 \mathrm{~Hz}-25 \mathrm{kHz})$ as determined through phase-noise sideband measurements over a series of frequency spans. The frequency structure of a laser's timing fluctuations is more compactly presented as a $\log -\log$ plot of the phase noise spectral density $L(f)$. Fig. 4 shows the phase noise spectrum $L_{1}(f)$ [calculated from phasenoise measurements at a high harmonic $N$ of the laser pulse repetition frequency through the relationship $L_{N}(f)$ $\left.=N^{2} L_{1}(f)\right]$ of a Quantronix $1.3 \mu \mathrm{m}, 100 \mathrm{MHz}$ secondharmonic mode-locked Nd: YAG laser. The rms jitter of this laser is 2.75 ps over the $(5 \mathrm{~Hz}-25 \mathrm{kHz})$ frequency range.

We are not aware of any extensive study in the literature concerning the origin of timing jitter in mode-locked lasers. Significant work has been published concerning the origins of phase noise in radio-frequency signal sources [16], including analyses [27] using the van der Pol [17] model of the harmonic oscillator. Insofar as the pulse repetition frequency of the laser intensity is synchronized to the cavity mode spacing through modulation of the cavity loss, mode-locked lasers are similar to electrical injection-locked oscillators [17]-[19] (it is to be emphasized that it is the oscillations in the mode-locked laser intensity envelope which are injection locked, and not the laser optical frequency [20]). An extensive analysis of phase noise in microwave injection-locked oscillators is given by $\mathrm{Ku}-$ rokawa [19]. If the oscillations in the mode-locked laser intensity were to be similarly represented through use of the canonical van der Pol model, then the phase noise arising from laser gain and resonator fluctuations could be calculated. Investigation of the origins of the laser timing fluctuations is beyond the scope of this text. The general

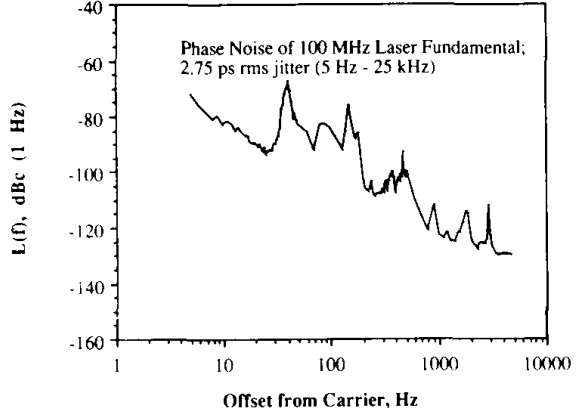

Fig. 4. Phase noise $L_{1}(f)$ of a Quantronix $1.3 \mu \mathrm{m}, 100 \mathrm{MHz}$, secondharmonic mode-locked Nd: YAG laser.

features of mode-locked laser phase-noise behavior are consistent with the injection-locked model; von der Linde [10] observed that the phase noise of a mode-locked argon laser decreased as the drive to the acoustooptic modulator was increased, while we have observed that Nd:YAG laser phase noise increases as the mode-locking frequency is detuned from the laser cavity frequency.

\section{Alternative Methods of Measurement}

Laser timing stability measurements can also be made by cross-correlation methods. The laser intensity autocorrelation is measured over a large range of correlation delays. For delays small in comparison to the laser pulse repetition period $T_{l}$, the single-pulse autocorrelation is measured and the laser pulsewidth is determined. For delays in the vicinity of $n T_{l}$, the autocorrelation is the cross correlation between pulses separated in time by $n T_{l}$; the width of the correlation spike in the vicinity of $n T_{l}$ is the sum of the single-pulse autocorrelation width and the mean-squared laser timing variation over the period $\Delta T$ $=n T_{l}$, as given by (8).

The cross correlation measures the change in laser timing over a period $\Delta T$ equal to the cross-correlation delay. Equivalently, jitter components with frequency above $\omega_{\text {low }}$ $=\pi / 2 \Delta T$ are measured. For example, a cross correlation using $1 \mu$ s delay measures timing jitter Fourier components with frequencies above $250 \mathrm{kHz}$. The required crosscorrelation delay should be equal to the duration of the period of observation over which laser timing variations are of concern. In an experiment where the timing fluctuations occurring over a period of $\sim 10 \mu \mathrm{s}$ duration are of interest, optical fiber delay-lines of $\sim 2 \mathrm{~km}$ length would be required for the cross correlation.

In many optical probing experiments [1]-[9], the detected signal-to-noise ratio is enhanced by signal averaging, and the duration of the experiment is often on the order of $1 \mathrm{~s}$ or longer. If such experiments are performed with the system under test excited and probed by separate but synchronized lasers or if the system under test is probed by a laser synchronized to a microwave excitation source, then the experimental time resolution is degraded in proportion to the variation in the laser timing over the duration of the experiment. In an experiment of $1 \mathrm{~s} \mathrm{du}$ - 
ration, the change in laser timing during a $1 \mathrm{~s}$ period is of concern; the required $1 \mathrm{~s}$ cross-correlation delay then stipulates a $\sim 2 \times 10^{8} \mathrm{~m}$ optical fiber delay line.

Phase-noise measurements can be made using precision phase detection systems [12]. Compared to direct spectral measurement (Section II), a phase detector provides larger discrimination between amplitude and phase fluctuations. If the discrimination is sufficient, phase-noise measurements can be made directly on the first harmonic of the laser pulse repetition frequency. For timing measurements of lasers with small timing jitter and large amplitude fluctuations, the phase versus amplitude discrimination of the phase detector may be insufficient; phase noise measurements should then be made a sufficiently high harmonic of the laser pulse repetition rate. In general, the discrimination against amplitude noise provided by the phase detector permits phase-noise measurements at a lower order laser harmonic then is required for direct spectral measurement of phase noise.

\section{Timing Stabilization by Feedback: Theory}

The pulse-timing fluctuations of a mode-locked $\mathrm{Nd}$ : Y AG laser can be reduced by a phase-lock-loop feedback system [21] (Fig. 5), as first demonstrated by Cotter [22]. A photodiode monitors the laser pulse train, and the phase of its fundamental component is compared to that of a very stable reference oscillator, generating a phase error signal. The signal driving the mode-locking acoustooptic (A-O) cell, whose frequency is half the laser pulse repetition frequency, is generated by frequency division from the reference oscillator; its timing (phase) is adjusted with a voltage-controlled phase shifter driven by the amplified and filtered (frequency-compensated) phase error signal. Given an error-free phase detector and a stable feedback loop with high loop transmission, the loop continuously adjusts the phase of the laser pulse train to equal the phase of the reference oscillator, suppressing the laser timing fluctuations. Jitter suppression is limited by spurious outputs from the phase detector, by the phase noise of the reference oscillator, and by the limited loop gain and limited loop bandwidth attainable in a stable, well-damped feedback control system. Spurious outputs from the phase detector include additive noise from various system components and inadvertent detection of amplitude noise by the phase detector. Amplitude noise sensitivity, similar to amplitude-noise interference in phase (frequency) modulation radio systems [23], is here referred to as amplitude modulation to phase modulation (AM-PM) conversion.

\section{A. AM-PM Conversion by Phase Detector DC Offset}

Radio-frequency phase detection can be performed using a double-balanced mixer [24] to generate the product of the reference signal and the signal of unknown phase (Fig. 6). The mixer output contains a dc phase term plus high-frequency components removed by loss-pass filtering. The output of the phase detector is a function of

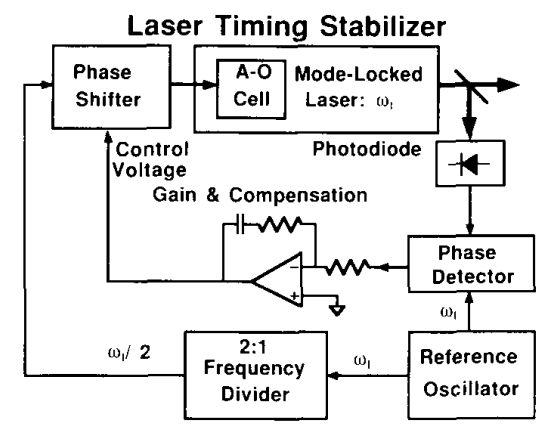

Fig. 5. Phase-lock loop for laser timing stabilization.

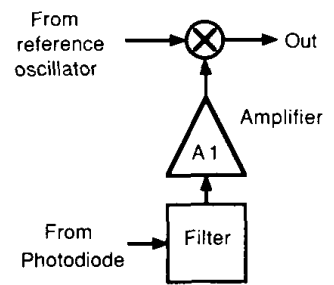

Fig. 6. Simple phase detector using a double-balanced mixer. The filter passes only the fundamental component at $\omega_{l}$ of the laser pulse train.

both laser phase and amplitude:

$$
V_{\text {detector }}=k_{\theta} \bar{P}[1+N(t)] \sin (\theta)+V_{o s}
$$

where $\theta=2 \pi\left[T_{0}+J(t)\right] / T$ is the phase difference (for convenience, a $\pi / 2$ phase factor has been dropped) and $V_{o s}$ is the dc offset voltage of the phase detector arising from mixer imbalance [24]. The detector phase coefficient is $k_{\theta} \bar{P}=A_{M 1} V_{\text {in }}$, where $A_{M 1}$ is the mixers' conversion efficiency in units of volts/volt. The peak voltage of the sinusoidal phase detector input voltage is $V_{\text {in }}=$ $A_{A 1} \sqrt{2 P_{\text {photodiode }} Z_{0}}$ where $A_{A 1}$ is the voltage gain of amplifier $A 1$ and $P_{\text {photodiode }}$ is the power of the fundamental component (at $\omega_{l}$ ) of the photodiode signal in a system with impedance $Z_{0}$ (usually $50 \Omega$ ).

For small $\theta$, the detector output is approximately

$$
V_{\text {detector }} \simeq k \bar{P}\left([1+N(t)] T_{0}+J(t)\right)+V_{o s}
$$

where $k=\omega_{l} k_{\theta}$. The terms in $N(t)$ in (10) result in sensitivity to laser amplitude noise. To assess this effect, assume that we have a feedback loop with large loop gain at all frequencies, forcing the phase detector output to zero. The laser timing becomes

$$
\begin{aligned}
T_{0} & =-V_{o s} / k \bar{P} \\
J(t) & =T_{0} N(t) .
\end{aligned}
$$

Thus, the dc offset voltage causes timing drift with changes in mixer offset and causes first-order variations in the phase detector output with laser amplitude fluctuations, producing residual closed-loop timing jitter (Fig. 7). Although signal limiters may be employed to maintain 


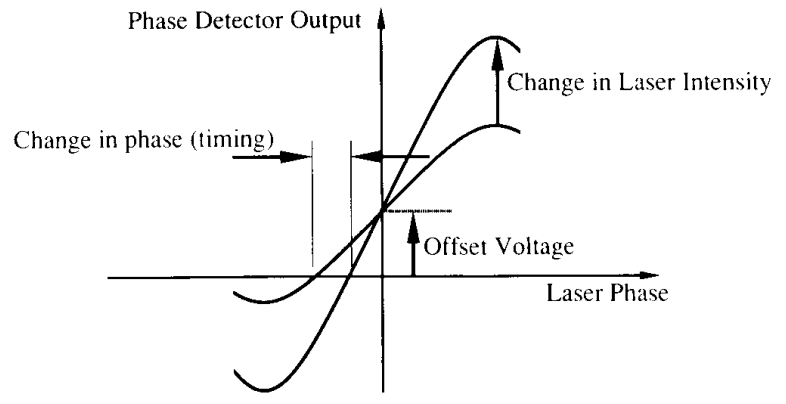

Fig. 7. AM-PM conversion by phase detector dc offset: in the presence of nonzero dc offset voltage, the amplitude variability of the phase-detector output causes an erroneous timing error signal. For clarity, the offset voltage is greatly exaggerated.

a constant phase-detector input power [22], limiters themselves cause AM-PM conversion, typically on the order of $0.1 \% \mathrm{~dB}$ change in the input power level. This is a factor of 5 poorer than that required for the timing stabilization system described in this text. In general, nonlinear processing of the photodiode signal may result in AMPM conversion. In any system used for laser timing measurement or picosecond laser synchronization, the AMPM conversion of system components such as mixers, amplifiers, limiters, comparators, and automatic-gaincontrol circuits must be evaluated.

An improved phase detector (Fig. 8) suppresses the effect of the mixer offset by increasing the slope coefficient $k \bar{P}$ of the detector. The technique is similar to that of chopper-stabilized dc amplifiers and synchronous ("lockin') signal detectors. The reference oscillator output is multiplied in a double-balanced-mixer $(M 1)$ by a squarewave phase-modulation signal whose frequency is $\omega_{\mathrm{IF}}$. After narrow-band filtration to remove spurious mixer outputs, the resulting reference signal is then a sine wave at the laser repetition frequency $\omega_{l}$ whose phase is inverted $+180^{\circ} /-0^{\circ}$ at the chopping rate $\omega_{\mathrm{IF}}$. The fundamental component at $\omega_{l}$ of the photodiode signal is passed to a second mixer (M2), in which the laser phase is compared to that of the phase-modulated reference signal. The output of the second mixer is then a spurious dc offset voltage $V_{o s, 2}$, plus a square wave at frequency $\omega_{\mathrm{IF}}$ whose amplitude varies in proportion to the phase difference between the photodiode and the reference signals. The square-wave is band-pass filtered, separating the desired signal at $\omega_{\mathrm{IF}}$ from undesired signals at dc $\left(V_{o s}\right)$ and multiples of $\omega_{\mathrm{IF}}$ and $\omega_{l}$, and is amplified by a gain $A_{A 2}$ on the order of $40 \mathrm{~dB}$. The phase-error signal, now a variableamplitude sine wave with frequency $\omega_{\mathrm{IF}}$, is synchronously detected by a third mixer ( $M 3$ ), producing an output voltage proportional to the laser phase. As with the simple phase detector, the chopper-stabilized detector has sensitivity to laser amplitude fluctuations and has a dc offset $V_{o s}^{\prime}$ arising from the third mixer:

$$
V_{\text {detector }}^{\prime}=k^{\prime} \bar{P}\left([1+N(t)] T_{0}+J(t)\right)+V_{o s}^{\prime} .
$$

In the chopper-stabilized detector, the phase-detector gain

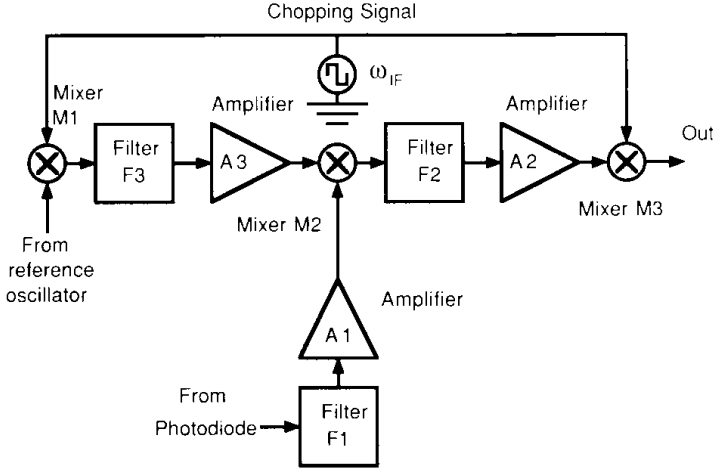

Fig. 8. Chopper-stabilized phase detector. See text for description of operation. $F 3$ is a filter with passband $-\omega_{l} \pm 5 \omega_{\mathrm{IF}}, F \mathrm{l}$ is a filter with passband $\sim \omega_{l} \pm \omega_{l} / 2$, and $F 2$ is a filter with passband $\sim \omega_{\mathrm{IF}} \pm \omega_{\mathrm{IF}} / 2$.

coefficient is given by

$$
k^{\prime} \bar{P}=\omega_{l} \sqrt{2 P_{\text {photodiode }} Z_{0}} A_{A 1} A_{M 2} A_{A 2} A_{M 3}\left(\frac{8}{\pi^{2}}\right)
$$

where $A_{A 2}$ is the voltage gain of amplifier $A 2$ and $A_{M 2}$ is the RF-IF conversion loss (in units of volts/volts) of mixer $M 2$. The factor of $\omega_{l}$ results from the conversion from phase sensitivity coefficient to timing sensitivity coefficient, and the factor of $8 / \pi^{2}$ is a consequence of chopping with a square-wave signal, but subsequently synchronously detecting only its sinusoidal fundamental component. With the chopper-stabilized phase detector, $\mathrm{dc}$ offset will result in timing offset and timing jitter given by

$$
\begin{aligned}
T_{0} & =-V_{o s}^{\prime} / k^{\prime} \bar{P} \\
J(t) & =T_{0} N(t) .
\end{aligned}
$$

The spectral density $S_{J, o s}(\omega)$ of spurious timing fluctuations resulting from detector dc offset will be

$$
S_{J . o s}(\omega)=T_{0}^{2} S_{N}(\omega) \text {. }
$$

\section{B. AM-PM Conversion Through Device Saturation}

The simplest method of attaining large phase detector sensitivity is to provide large input signal levels to the detector. Problems with dc offsets are reduced, as are the effects of additive noise voltages and noise currents within the feedback loop. Above some power level, nonlinear effects within both the amplifier $A 1$ and the mixer $M 2$ used for phase detection will result in phase modulation of the photodiode signal in proportion to the signal's amplitude modulation (AM-PM conversion). AM-PM conversion results from cubic terms in the transfer characteristics of a device; the effect is similar to self-phase modulation in optical fibers. As the timing stabilization system is unable to distinguish the laser's timing fluctuations from spurious phase modulation of the photodiode signal, AM-PM conversion results in timing fluctuations of the stabilized laser, occurring in proportion to the laser intensity fluctuations. 
Given AM-PM conversion, laser timing fluctuations occur despite a near-zero output from the stabilizer's phase detector; in an operating laser timing stabilization system, the presence of significant AM-PM conversion can be inferred by measuring the phase-detector output signal and comparing its magnitude to the measured laser timing jitter. Assuming insignificant AM-PM conversion and insignificant detector noise (Section II-C), the rms laser timing jitter can be calculated from the rms phase detector output signal through $V_{\text {detector }}=k \bar{P} J(t)$. A phase-detector output signal small in comparison to the timing jitter determined through phase-noise measurements is indicative of either phase-detector AM-PM conversion or phase-detector additive noise.

The response of a weakly nonlinear system can be described by a frequency-domain Volterra series expansion [25], [26]. If the input to the system is small (and this is required in order to have adequately small AM-PM conversion), the Volterra series can be truncated to third order with sufficient accuracy. Furthermore, second-order terms in the Volterra series result in nonlinear responses at $\mathrm{dc}$ and twice the input frequency; as these will be outside the bandwidth of various filters within the system, their effect can be neglected. The nonlinear response of amplifiers and mixers within the timing stabilizer is then modeled as

$$
\begin{aligned}
V_{\text {out }}(\omega) \simeq & H_{1}(\omega) V_{\text {in }}(\omega)+\frac{1}{4 \pi^{2}} \iint_{-\infty}^{+\infty} \\
& \cdot H_{3}\left(\Omega_{1}, \Omega_{2}, \omega-\Omega_{1}-\Omega_{2}\right) \times V_{\text {in }}\left(\Omega_{1}\right) \\
& \cdot V_{\text {in }}\left(\Omega_{2}\right) V_{\text {in }}\left(\omega-\Omega_{1}-\Omega_{2}\right) d \Omega_{1} d \Omega_{2} .
\end{aligned}
$$

Consider the response of a third-order nonlinear system to an input signal $v_{\text {in }}(t)$ with small $\left(N_{c}<1\right)$, narrowband amplitude modulation $\left(\omega_{m}<\omega_{l}\right)$ :

$$
v_{\text {in }}(t)=v_{0}\left(1+N_{c} \cos \omega_{m} t\right) \cos \omega_{l} t .
$$

Because $\omega_{m} \ll \omega_{l}$, and because of symmetries, the relevant Volterra coefficients in (16) reduce to two parameters, $H_{1}$ and $H_{3}$ :

$$
\begin{aligned}
& H_{1}\left( \pm \omega_{l} \pm \omega_{m}\right)=H_{1}\left( \pm \omega_{l}\right) \\
& H_{3}\left( \pm \omega_{l} \pm \omega_{m}, \pm \omega_{l} \pm \omega_{m}, \pm \omega_{l} \pm \omega_{m}\right) \\
& \quad \simeq H_{3}\left( \pm \omega_{l}, \pm \omega_{l}, \pm \omega_{l}\right) \\
& H_{1}\left( \pm \omega_{l} \pm \omega_{m}\right) \simeq H_{1}\left( \pm \omega_{l}\right) \\
& H_{3}\left( \pm \omega_{l} \pm \omega_{m}, \pm \omega_{l} \pm \omega_{m}, \pm \omega_{l} \pm \omega_{m}\right) \\
& \quad \simeq H_{3}\left( \pm \omega_{l}, \pm \omega_{l}, \pm \omega_{l}\right) \\
& H_{1}=H_{1}\left(\omega_{l}\right)=H_{1}^{*}\left(-\omega_{l}\right) \\
& H_{3}=H_{3}\left(\omega_{l}, \omega_{l},-\omega_{l}\right)=H_{3}\left(\omega_{l},-\omega_{l}, \omega_{l}\right) \\
& \quad=H_{3}\left(-\omega_{l}, \omega_{l}, \omega_{l}\right) \\
& \quad=H_{3}^{*}\left(-\omega_{l},-\omega_{l}, \omega_{l}\right)=H_{3}^{*}\left(-\omega_{l}, \omega_{l},-\omega_{l}\right) \\
& =H_{3}^{*}\left(\omega_{l},-\omega_{l},-\omega_{l}\right) .
\end{aligned}
$$

Given a third-order nonlinear system with an amplitudemodulated input as in (17), (16), (18) permit calculation of the output spectrum. Additional sidebands at $\left(\omega_{l} \pm \omega_{m}\right)$ arise from the cubic nonlinear term through three-frequency mixing, $\omega_{l}+\omega_{l}-\left(\omega_{l} \mp \omega_{m}\right)$, and permutations. The resulting phase modulation is calculated from the phase of these spurious sidebands using (3), (4). The output voltage $v_{\text {out }}(t)$ is phase modulated by $\theta_{c} \cos \omega_{m} t$ where

$$
\frac{\theta_{c}}{N_{c}}=\frac{9}{4} v_{0}^{2} \mathfrak{J}\left(\frac{H_{3}}{H_{1}}\right) .
$$

$\mathfrak{I}$ denotes the imaginary part of a complex number. The AM-PM conversion factor $\mathfrak{I}\left(H_{3} / H_{1}\right)$ is rarely specified for an electronic component. An upper bound can be found given knowledge of the (input-referred) two-tone thirdorder intercept power $P_{3 o i}$, a parameter commonly used to specify the relative level of third-order nonlinearities in microwave components [25]. This is

$$
P_{3 o i}=\left\|\frac{H_{1}}{H_{3}}\right\| \frac{2}{3 Z_{0}}
$$

where $Z_{0}$ is the system impedance, usually $50 \Omega$. The spurious phase modulation arising from an amplitude-modulated input can thus be bounded by

$$
\left\|\frac{\theta_{c}}{N_{c}}\right\| \leq \frac{9 v_{0}^{2}}{4}\left\|\frac{H_{3}}{H_{1}}\right\|=\frac{3 v_{0}^{2}}{2 P_{3 o i} Z_{0}} .
$$

The spurious phase modulation occurring in amplifier $A 1$ and mixer $M 2$ will result in erroneous phase error signals at the output of the phase detector. With the approximation of large loop gain, the feedback loop will adjust the laser timing to force the phase detector output to zero, and residual timing fluctuations then result from AM-PM conversion. If the power spectral density of the residual closed-loop timing jitter due to AM-PM conversion through third-order nonlinearities is denoted as $S_{J, 3 o}(\omega)$, it is found that

$$
S_{J, 3 o}(\omega) \leq \frac{9}{\omega_{l}^{2}}\left(\frac{P_{\omega l}}{P_{3 o i}}\right)^{2} S_{N}(\omega)
$$

where $P_{\omega l}=v_{0}^{2} / 2 Z_{0}$ is the signal power ( at the frequency $\omega_{l}$ ) input to the device under consideration.

\section{Additive Noise in the Phase Detection System}

For a laser with subpicosecond timing jitter and a $\sim 100$ $\mathrm{MHz}$ pulse repetition rate, the phase noise sidebands associated with the laser fundamental will have very low power. If the phase detector additive noise has power comparable to the power of these sidebands, significant spurious phase detector outputs will arise. The noise figure [27] of the phase detection system is

$$
\begin{aligned}
F_{\text {detector }}= & F_{A 1}+\frac{F_{M 2}-1}{A_{A 1}^{2}}+\frac{F_{A 2}-1}{A_{A 1}^{2} A_{M 1}^{2}\left(8 / \pi^{2}\right)} \\
& +\frac{F_{M 3}-1}{A_{A 1}^{2} A_{M 1}^{2}\left(8 / \pi^{2}\right) A_{A 2}^{2}}
\end{aligned}
$$


where $F_{A 1}, F_{A 2}, F_{M 2}$, and $F_{M 3}$ are the noise figures of the amplifiers $A 1$ and $A 2$ and mixers $M 2$ and $M 3$. As with (13), the factors of $8 / \pi^{2}$ in (23) are due to synchronous detection of only the sinusoidal fundamental component of a square-wave chopping signal. The phase detector noise can be decomposed through (4) into spurious amplitude-noise and phase-noise sidebands of equal power. Assuming large feedback loop gain, so that loop adjusts the laser timing to force the phase detector output to zero, the phase detector noise will result in a residual closedloop timing jitter with spectral density

$$
S_{J \text {, detector }}(\omega)=\frac{k T F_{\text {detector }}}{2 \omega_{l}^{2} P_{\text {photodiode }}}
$$

where $k$ is Boltzmann's constant and $T$ is the temperature.

The gain and compensation amplifier also has some equivalent input noise voltage with spectral density $S_{V, \text { amp }}(\omega)$, which will result in residual closed-loop jitter having a power spectral density

$$
S_{J, \text { amp }}(\omega)=\frac{S_{V, \text { amp }}(\omega)}{\left(k^{\prime} \bar{P}\right)^{2}} .
$$

To suppress these residual noise terms, the signal levels should be as large as possible (as limited by AM-PM conversion effects), and the components should be selected for low noise.

\section{Reference Oscillator Phase Noise}

A final error term in the phase detection system is the phase noise (timing jitter) of the reference oscillator; in an ideal timing stabilization system, the laser phase will track that of the reference. Moderate-cost $\sim 100 \mathrm{MHz}$ quartz crystal oscillators are available with phase noise spectral densities $L(f)$ on the order of $-100 \mathrm{dBc}(1 \mathrm{~Hz})$ at $f=10 \mathrm{~Hz},-130 \mathrm{dBc}(1 \mathrm{~Hz})$ at $f=100 \mathrm{~Hz},-145$ $\mathrm{dBc}(1 \mathrm{~Hz})$ at $f=1 \mathrm{kHz}$, and $-150 \mathrm{dBc}(1 \mathrm{~Hz})$ at $f=$ $10 \mathrm{kHz}$ [28]. If the oscillation frequency must be tuned to accommodate variations in the laser cavity frequency, then a frequency synthesizer having comparable phase noise can be used. These are expensive $(\sim 30000)$.

\section{E. Loop Bandwidth and Stability}

Given a phase detector with negligible spurious outputs, the timing jitter suppression is set by the feedback loop transmission $G(\omega)$ :

$$
\begin{aligned}
S_{\text {closed loop }}(\omega) & =\left\|\frac{1}{1+G(\omega)}\right\|^{2} S_{\text {open loop }}(\omega), \\
G(\omega) & =k^{\prime} \bar{P} A_{\text {comp }}(\omega) k_{s} H_{L}(\omega) .
\end{aligned}
$$

$A_{\text {comp }}(\omega)$ is the transfer function of the amplifier providing loop gain and compensation, $k_{s}$ is the delay coefficient of the voltage-controlled phase shifter (i.e., $k_{s}=$ $d \tau / d V_{\text {control }}$, where $\tau$ is the delay and $V_{\text {control }}$ is the control voltage ), and $H_{L}(\omega)$ is the phase transfer function of the laser.
Selection of the gain and compensation $A_{\text {comp }}(\omega)$ to attain the desired loop characteristics is the subject of control system theory [29]. Large $G(\omega)$, desirable at all frequencies at which $S_{\text {open loop }}(\omega)$ is significant, is limited by stability constraints arising from $H_{L}(\omega)$. If the feedback loop is opened (this is achieved in practice by leaving the loop active, but decreasing the loop bandwidth to $\sim 1 \mathrm{~Hz}$ ) and the phase of the drive signal to the acoustooptic cell is perturbed by applying a step-function drive signal to the voltage-controlled phase shifter, then the output of the phase detector will show the transient behavior of the laser timing in response to a step-function phase shift. For our Nd: YAG laser (Fig. 9), this step response conforms well to $h_{l}(t)=u(t)\left(1-e^{-t / \tau_{p}}\right), \tau_{p}=140 \mu \mathrm{s}$ where $u(t)$ denotes the unit step function. The laser phase transfer function is therefore

$$
H_{L}(\omega) \simeq \frac{1}{1+j \omega / \omega_{p}}
$$

where $\omega_{p}=1 / \tau_{p}$; for our laser, $\omega_{p} / 2 \pi \sim 1.1 \mathrm{kHz}$.

The loop bandwidth (the frequency at which $\|G\|=$ 1 ) must be constrained, due to additional poles at higher frequencies arising from either the laser or the electronics. Simple feedback systems commonly use an integration in the feedback loop. In this case, $A_{\text {comp }}(\omega)=\omega_{c} / j \omega$, and the loop-noise suppression factor $R(\omega)$ is given by

$$
R(\omega) \equiv \frac{1}{1+G(\omega)}=\frac{j \omega\left(2 \zeta / \omega_{n}\right)-\omega^{2} / \omega_{n}^{2}}{1+j \omega\left(2 \zeta / \omega_{n}\right)-\omega^{2} / \omega_{n}^{2}}
$$

where the loop natural frequency is $\omega_{n}=\left(k^{\prime} \bar{P} k_{s} \omega_{c} \omega_{p}\right)^{1 / 2}$ and the loop damping is $\zeta=\left(\omega_{p} / 4 k^{\prime} \bar{P} k_{s} \omega_{c}\right)^{1 / 2}$. If an attempt is made to attain large loop gain and large loop bandwidth by choosing a large gain coefficient $k^{\prime} \bar{P} k_{s} \omega_{c}$, the loop damping factor $\zeta$ will be degraded. For $\zeta<$ $1 / \sqrt{2}$, the spectral density $S_{J}(\omega)$ of the timing jitter will be increased for frequencies near $\omega_{n}$. For a well-damped control system without peaking of the closed-loop $S_{J}(\omega)$ near $\omega_{n}$, the loop damping $\zeta$ must be greater than $1 / \sqrt{2}$ and the loop natural frequency is then bounded: $\omega_{n} \leq$ $\omega_{p} / \sqrt{2}$. Thus, in a simple control system using integrating feedback, the laser pole frequency limits the suppression of timing jitter to those frequency components below $\omega_{p} / \sqrt{2}$. Even within this bandwidth, the phase-noise suppression is finite, as given by (26). Unfortunately, $S_{J}(\omega)$ has significant energy at frequencies approaching the laser pole frequency $\omega_{p}$.

Well-damped control loops with larger loop bandwidth can be attained by a variety of compensation techniques [29]. Compensator design is guided by the sensitivity of the resulting system characteristics to changes in the laser parameters. Simple lead compensation permits a moderate increase in loop bandwidth. A compensating zero is introduced:

$$
A_{\text {comp }}(\omega)=\frac{\omega_{c}}{j \omega}\left(1+j \omega / \omega_{p}\right)=\frac{\omega_{c}}{j \omega}+\frac{\omega_{c}}{\omega_{p}},
$$




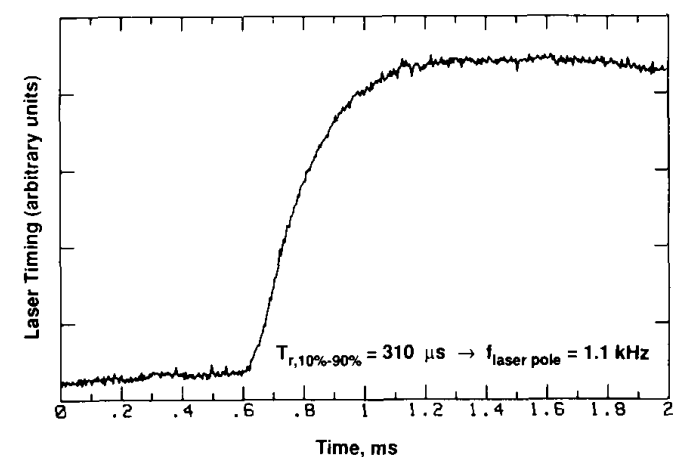

Fig. 9. Transient response of the phase of the Spectra-Physics Nd: YAG laser to a step change in the phase of the mode-locking drive signal. The response is that of a first-order dynamic system with a $1.1 \mathrm{kHz}$ pole.

and the loop suppression becomes

$$
R(\omega)=\frac{j \omega / \omega_{\text {loop }}}{1+j \omega / \omega_{\text {loop }}} \quad \text { where } \omega_{\text {loop }}=k^{\prime} \bar{P} k_{s} \omega_{c}
$$

The feedback loop is well damped, even for loop bandwidths $\omega_{\text {loop }}$ exceeding $\omega_{p}$.

\section{IMPLEMENTATION}

We have stabilized the timing fluctuations of an $82 \mathrm{MHz}$ CW mode-locked Nd: YAG laser, Spectra-Physics series 3000. As the laser system is used in picosecond electrooptic sampling experiments, the Nd:YAG laser output is compressed to 1.25 ps FWHM using a fiber-grating pulse compressor [30].

To facilitate compression to $1.25 \mathrm{ps,} \mathrm{the} \mathrm{standard}$ acoustooptic mode locker was replaced by an experimental Spectra-Physics unit, resulting in a reduction of the laser output pulsewidth from 90 to $\sim 45 \mathrm{ps}$ FWHM. The unstabilized phase noise sidebands have significant spectral density up to a frequency of $-5 \mathrm{kHz}$, and a similar feedback-loop bandwidth is thus required. Given significant phase noise over a $\pm 5 \mathrm{kHz}$ bandwidth, after stabilization the spectral density $L_{1}(f)$ must be suppressed to approximately $-116 \mathrm{dBc}(1 \mathrm{~Hz})$ over most of this bandwidth if $\sim 300 \mathrm{fs}$ timing fluctuations are to be attained. The stabilizer must suppress the phase noise to $L_{1}(f) \sim$ $-116 \mathrm{dBc}(1 \mathrm{~Hz})$ in the presence of much larger laser amplitude noise $S_{N}(f) \simeq-85 \mathrm{dBc}(1 \mathrm{~Hz})$ at $f=100$ $\mathrm{Hz}$ and $-95 \mathrm{dBc}(1 \mathrm{~Hz})$ at $f=1 \mathrm{kHz}$.

The timing stabilization system has been implemented following the design considerations outlined in Section IV. The phase detection system is chopper stabilized as in Section IV-A, with chopping at $1 \mathrm{MHz}$; the phase detector slope $k^{\prime} \bar{P}$ is $1 \mathrm{~V} / \mathrm{ns}$, and the mixer offset is less than $1 \mathrm{mV}$, resulting in a static timing offset $T_{0}$ of less than 1 ps. By (15), the resulting AM-PM conversion due to dc offset is suppressed by $-66 \mathrm{~dB}$, i.e., offset-induced AM-PM conversion will result in single-sideband phase noise $L_{1}(f) 66 \mathrm{~dB}$ less than the laser amplitude noise $S_{N}(f)$. By setting the input power levels to amplifier $A 1$ and mixer $M 2$ more than $35 \mathrm{~dB}$ below the third-order in-

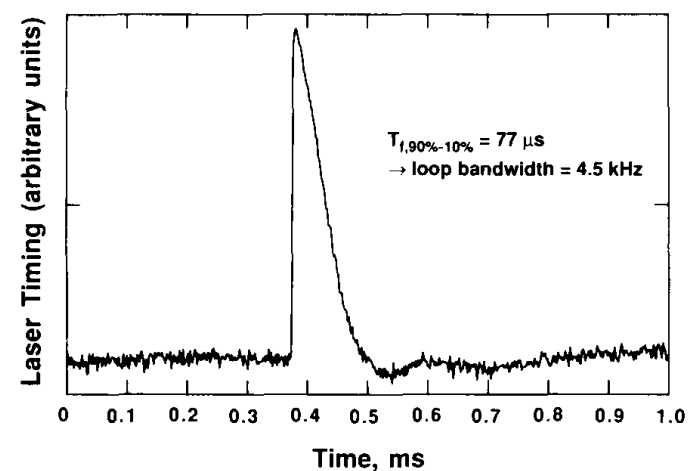

Fig. 10. Suppression of a step-function error signal injected into the phaselock loop. From this measurement, the loop bandwidth $f_{\text {loop }}=4.4 \mathrm{kHz}$ and loop damping $\zeta \simeq 0.7$ are determined.

tercept power levels of these components, AM-PM conversion due to third-order products is suppressed by greater than $60 \mathrm{~dB}$, as calculated by (25). The noise figure $F_{\text {detector }}$ of the phase detector is $10.5 \mathrm{~dB}$, and the photodiode power $P_{\text {photodiode }}$ at the laser repetition frequency $\omega_{l}$ is $-24 \mathrm{dBm}$; hence, the phase detector noise will result in a spurious phase noise spectral density of $-140 \mathrm{dBc}$ $(1 \mathrm{~Hz})$.

The reference oscillator for the timing stabilization system is a Hewlett-Packard 8662A low phase-noise frequency synthesizer. No phase-noise specification is stated for this synthesizer operating at $82 \mathrm{MHz}$, but over the $320-640 \mathrm{MHz}$ range, the phase noise specification is $L(f) \leq-104 \mathrm{dBc}(1 \mathrm{~Hz})$ at $100 \mathrm{~Hz},-121 \mathrm{dBc}(1 \mathrm{~Hz})$ at $1 \mathrm{kHz}$, and $-131 \mathrm{dBc}(1 \mathrm{~Hz})$ at $10 \mathrm{kHz}$. Extrapolation to $82 \mathrm{MHz}$ is uncertain, but it is likely that the oscillator's timing deviations are relatively independent of oscillation frequency, in which case phase noise power will scale as the square of oscillation frequency. The phase noise of the HP8662A at $82 \mathrm{MHz}$ is then estimated as $-116 \mathrm{dBc}$ $(1 \mathrm{~Hz})$ at $100 \mathrm{~Hz},-133 \mathrm{dBc}(1 \mathrm{~Hz})$ at $1 \mathrm{kHz}$, and -143 $\mathrm{dBc}(1 \mathrm{~Hz})$ at $10 \mathrm{kHz}$. Given both the small difference between the estimated reference phase noise and the objective stabilized laser phase noise and the uncertainty of the estimate, these data will have to be verified if the laser phase noise is to be further reduced.

The control loop bandwidth is set at approximately 5 $\mathrm{kHz}$; the loop is lead compensated, with a compensating zero at $1.1 \mathrm{kHz}$ cancelling the response of the $1.1 \mathrm{kHz}$ laser pole. Due to variability in the laser pole frequency, the compensation does not consistently provide good loop damping if the loop bandwidth is increased much beyond $7 \mathrm{kHz}$. The control loop dynamics are verified by measuring the suppression of a step-function error signal injected into the loop (Fig. 10); from this measurement, a damping factor of $\zeta \simeq 0.7$ and a loop bandwidth of $\sim 4.5$ $\mathrm{kHz}$ are determined. With these parameters set, the timing stabilization system should show significant phasenoise suppression over a $\pm 4.5 \mathrm{kHz}$ bandwidth, with only small residual phase noise arising from phase-detector spurious signals. 


\section{RESUlts}

Phase-noise measurements using a microwave spectrum analysis system (Fig. 11) were used to determine the laser timing jitter both before and after stabilization. The timing stabilizer monitors the pulse timing at the output of the Nd:YAG laser, and not at the output of the fibergrating pulse compressor. Therefore, any potential timing fluctuations arising in the fiber-grating compressor are not suppressed by the stabilizer. If the stabilizer instead monitored the output of the pulse compressor, the timing fluctuations arising in the fiber-grating compressor would also be suppressed in proportion to the feedback loop gain. This configuration is undesirable due to the resulting variations in the stabilizer loop gain: fiber polarization drift and the polarization sensitivity of the diffraction grating cause significant variations in the compressor output power.

While the pulse timing is stabilized at the $\mathrm{Nd}$ : YAG laser output, the timing jitter is measured at the output of the compressor. Timing fluctuations occurring in the fiber-grating pulse compressor are thus included in the measurement. The short pulse duration, and hence the wide frequency spectrum at the compressor output, also facilitate phase-noise measurements at very high harmonics of the pulse repetition rate.

As microwave spectrum analyzers are not generally available with the required $\sim 1 \mathrm{~Hz}$ resolution, the microwave spectrum analysis system (Fig. 11) uses a $0-20 \mathrm{GHz}$ microwave synthesizer (Hewlett-Packard HP8340B) as a local oscillator, and a $0-100 \mathrm{kHz}$, millihertz-resolution fast-Fourier-transform spectrum analyzer (Hewlett-Packard HP3561A). The laser system output is monitored by a $\sim 10 \mathrm{GHz}$ photodiode, with sufficient incident optical power to develop $\sim-40 \mathrm{dBm}$ photodiode output power at microwave-frequency harmonics of $\omega_{l}$. The photodiode signal is then amplified ( $15 \mathrm{~dB}$ gain, $13 \mathrm{~dB}$ noise figure) and filtered to reject low-frequency photodiode spectral content within the passband of the Fourier analyzer. The microwave synthesizer is locked to the $10 \mathrm{MHz}$ crystal oscillator frequency standard from the stabilizer's reference oscillator. The filter output at $n \omega_{l}$ is mixed against the microwave synthesizer output at $n \omega_{l}+50 \mathrm{kHz}$, translating the spectral line (and associated phase-noise sidebands) at $n \omega_{l}$ to the $50 \mathrm{kHz}$ difference frequency. The spectrum at the difference frequency is measured by the Fourier analyzer, and transferred to a computer for data analysis.

Fig. 12 shows the suppression of the laser phase noise $L_{1}(f)$ by the laser timing stabilizer, as measured at the pulse compressor output. To satisfy the small-angle requirement necessary for validity of (6), the phase noise data for the unstabilized laser was determined through sideband measurements $L_{36}(f)$ at a relatively low $3 \mathrm{GHz}$ harmonic of the laser pulse repetition rate. At $3 \mathrm{GHz}$, unstabilized phase-noise sidebands dominate the amplitudenoise sidebands. As the timing jitter of the stabilized laser is small, amplitude-noise sidebands dominate the stabi-

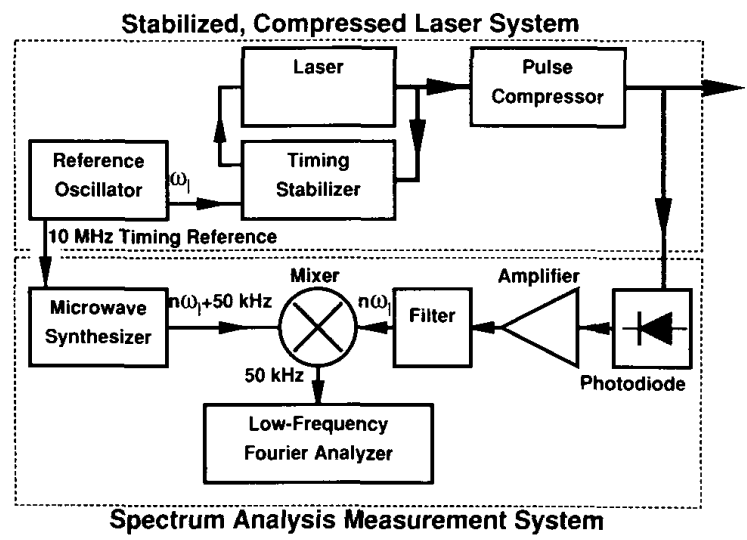

Fig. 11. Block diagram of stabilized, compressed laser system and the spectrum analysis system used for phase-noise measurements.

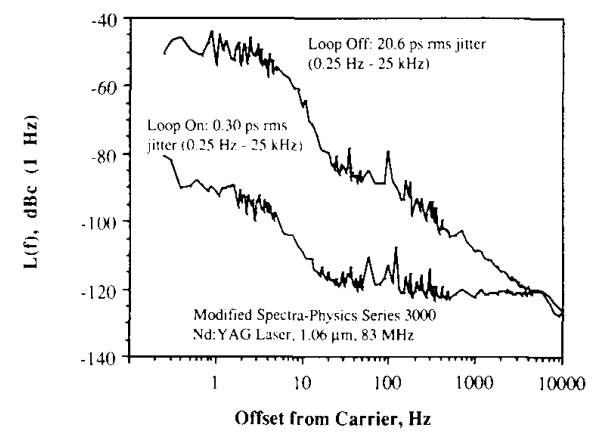

Fig. 12. Phase-noise spectral density, stabilizer on and off, as measured at the output of the pulse compressor. With the stabilizer on, phase-noise measurements below $30 \mathrm{~Hz}$ are dominated by amplitude noise.

lized phase-noise sidebands for harmonics below $\sim 5$ $\mathrm{GHz}$. The stabilized phase-noise data were thus determined by measurements $L_{200}(f)$ of the laser's 200th harmonic $(16.6 \mathrm{GHz})$. Even at this harmonic, sideband components below $30 \mathrm{~Hz}$ are dominated by amplitude noise, and the measured closed-loop $L_{1}(f)$ data of Fig. 12 is only an upper bound for $f \leq 30 \mathrm{~Hz}$.

Fig. 13 compares the calculated and measured performance of the timing stabilizer. At frequencies above $\sim 100 \mathrm{~Hz}$, the measured phase noise $L_{1}(f)$ is within 2 $\mathrm{dB}$ of that calculated by the loop rejection equation, (26). The residual stabilized phase noise arising from phase detector noise and AM-PM conversion effects is negligible above $30 \mathrm{~Hz}$, but may limit the stabilized phase noise below $\sim 10 \mathrm{~Hz}$. As the measured noise is amplitude-noise background in the phase-noise measurement, the phase noise and its limitations cannot be determined at frequencies below $30 \mathrm{~Hz}$. Integrating the measured phase-noise spectral densities of Fig. 12, the timing jitter of the laser is determined:

$$
\sigma_{J}= \begin{cases}20.6 \mathrm{ps}, & \text { open loop, } 0.25 \mathrm{~Hz}-25 \mathrm{kHz} \\ \leq 0.30 \mathrm{ps}, & \text { closed loop, } 0.25 \mathrm{~Hz}-25 \mathrm{kHz} .\end{cases}
$$

The reduction in phase noise is greater than $68: 1$ over the $0.25 \mathrm{~Hz}-25 \mathrm{kHz}$ frequency range. 


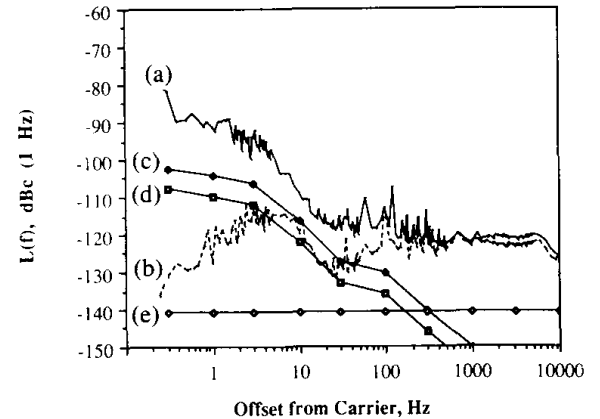

Fig. 13. Comparison of measured and calculated stabilized phase noise: (a) measured phase noise, (b) phase noise calculated by (26), (c) AMPM conversion through third-order products, (d) AM-PM conversion through dc offset, (e) phase detector noise.

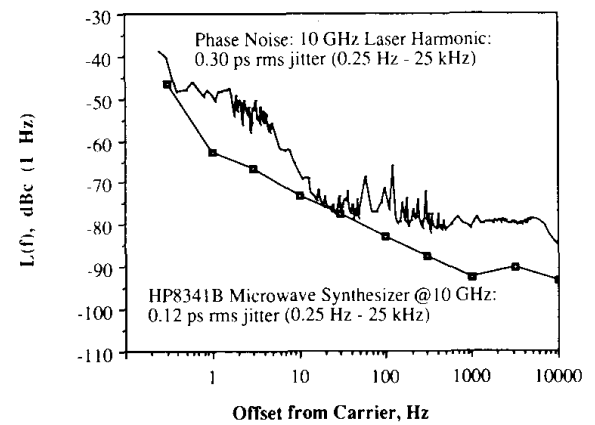

Fig. 14. Stabilized phase noise $L_{120}(f)$ of the $10 \mathrm{GHz}$ laser harmonic compared to the residual phase noise of a high-stability microwave synthesizer.

Fig. 14 compares the stabilized laser phase noise $L_{120}(f)$ of the $10 \mathrm{GHz}$ laser harmonic to the residual phase noise of the HP8340B microwave synthesizer operating at $10 \mathrm{GHz}$. The synthesizer phase noise was determined by spectrum analysis of the $50 \mathrm{kHz}$ difference frequency generated by mixing the $10 \mathrm{GHz}$ output of the HP8340B synthesizer with a $10.000,050 \mathrm{GHz}$ signal from a similar (Hewlett-Packard HP8341A) microwave synthesizer locked to the same $10 \mathrm{MHz}$ timing standard. As the measured phase noise is the sum of the two synthesizers' noise, the phase-noise power spectrum was corrected by $3 \mathrm{~dB}$. The rms timing jitter of the microwave synthesizer is $0.12 \mathrm{ps}$ over the $0.25 \mathrm{~Hz}-25 \mathrm{kHz}$ frequency range, a factor of 2.5 lower than the (measured) stabilized laser timing jitter.

It is to be emphasized that the data of Figs. 12, 13, and 14 are all residual phase-noise measurements, i.e., the relative phase (timing) fluctuations of two systems operating from the same crystal-oscillator fundamental timing reference. The absolute phase noise, i.e., the relative phase (timing) fluctuations of two systems locked to independent crystal oscillators, will be much larger due to crystal-oscillator $\sim 1 / f^{3}$ low-frequency phase noise. Because the laser timing stabilizer's reference oscillator can be locked to a crystal-oscillator timing reference shared with other lasers and microwave sources, residual phase noise is the relevant parameter.

\section{CONCLUSIONS}

In a frequency range of $(0.25 \mathrm{~Hz}-25 \mathrm{kHz})$, corresponding to a $1 \mathrm{~s}$ observation period, the timing fluctuations of a mode-locked laser have been reduced by greater than $68: 1$, attaining less than $0.30 \mathrm{ps}$ jitter. These timing fluctuations are less than three parts in $10^{5}$ of the laser pulse repetition period, while the amplitude fluctuations of the laser are approximately 1-2 percent of the average laser intensity. Stabilization of the timing to these levels requires a precise phase detector whose sensitivity to laser timing is much greater than its sensitivity to laser amplitude. The spectral density of the stabilized phase noise sidebands is approximately $-120 \mathrm{~dB}(1 \mathrm{~Hz})$ with respect to the $82 \mathrm{MHz}$ laser fundamental; as the thermal noise power power has spectral density $k T=-174 \mathrm{dBm}$ $(1 \mathrm{~Hz})$, the radio-frequency signal levels must be selected so that the associated phase-noise sidebands have power well above $k T F$ where $F$ is the noise figure of the phase detection system. The bandwidth of the unstabilized phase noise is significant in comparison to the bandwidth of the laser's dynamic phase response, and the feedback loop must be appropriately compensated in the loop is not to show instabilities or weakly damped resonances with associated peaking of the phase noise.

With these results, the timing stability of the $\mathrm{Nd}: \mathrm{YAG}$ laser is within an order of magnitude of that of high-stability microwave sources. The timing fluctuations are comparable to the pulsewidths generated by fiber-grating compressors and dye lasers pumped by $\mathrm{Nd}$ : YAG lasers, permitting picosecond physical experiments using multiple lasers and multiple electronic sources.

\section{ACKNOWLEDGMENT}

We thank T. Baer and J. Kafka (Spectra-Physics, Inc.) for aid in modification of the laser and guidance in operation of the pulse compressor. J. Bowers (then of AT\&T Bell Laboratories) provided the $10 \mathrm{GHz}$ photodiode. We acknowledge the generous equipment donations of Tektronix, Inc. and the Hewlett-Packard Company, and helpful discussions concerning timing stabilization with $\mathrm{T}$. Baer, B. Kolner (Hewlett-Packard Laboratories), and U. Keller (Stanford).

\section{REFERENCES}

[1] J. A. Valdmanis, G. A. Mourou, and C. W. Gabel, "Picosecond electro-optic sampling system," Appl. Phys. Lett., vol. 41, 211-212, 1982.

[2] J. A. Valdmanis and G. Mourou, "Subpicosecond electrooptic sampling, Principles and applications," IEEE J. Quantum Electron., vol. QE-22, pp. 69-78, 1986.

[3] B. H. Kolner and D. M. Bloom, "Electrooptic sampling in GaAs integrated circuits," IEEE J. Quantum Electron., vol. QE-22, pp. 79-93, 1986.

[4] K. W. Weingarten, M. J. W. Rodwell, and D. M. Bloom, "Picosecond optical sampling of GaAs integrated circuits," IEEE J. Quantum Electron., vol. 24 , pp. 198-220, Feb. 1988.

[5] P. G. May, J. M. Halbout, and G. L.-T. Chiu, "Noncontact highspeed waveform measurements with the picosecond photoelectron scanning electron microscope," IEEEJ. Quantum Electron., vol. 24, pp. 234-239, Feb. 1988.

[6] J. Bokor, R. Haight, J. Stark, R. H. Storz, R. R. Freeman, and P. H. Bucksbaum, "Picosecond time-resolved photoemission study of 
the InP (100) surface," in Picosecond Electronics and Optoelectronics, G. A. Mourou, D. M. Bloom, and C.-H. Lee, Eds. New York: Springer-Verlag, 1985, pp. 94-96

[7] D. H. Auston, K. P. Cheung, J. A. Valdmanis, and D. A. Kleinman, "Cherenkov radiation from femtosecond optical pulses in electrooptic media,"' Phys. Rev. Lett., vol. 53, pp. 1555-1558, 1984.

[8] J. M. Weisenfeld, R. S. Tucker, P. M. Downey, and J. E. Bowers, "Optical correlation measurements of switching transients in highspeed semiconductor lasers,"' Electron. Lett., vol. 22, pp. 396-397, 1986.

[9] J. A. Kash, J. C. Tsang, and J. M. Hvam, "Subpicosecond Raman spectroscopy of electron-LO phonon dynamics in GaAs," in Picosecond Electronics and Optoelectronics, G. A. Mourou, D. M. Bloom, and C.-H. Lee, Eds. New York: Springer-Verlag, 1985, pp. 87-90.

[10] D. von der Linde, "Characterization of the noise in continuously operating mode-locked lasers,"' Appl. Phys. B, vol. 39, pp. 201-217, 1986.

[11] J. Kluge, Ph.D. dissertation, Universität Essen, Essen, Federal Republic of Germany, 1984.

[12] "Phase noise characterization of microwave oscillators, phase detector method," Product Note 11729B-1, Hewlett-Packard Co., P.O. Box 10301, Palo Alto, CA 94303.

[13] "Measuring phase noise with the HP 3585A spectrum analyzer," Application Note AN 246-2, Hewlett-Packard Co., P.O. Box, 10301 Palo Alto, CA 94303.

[14] "Phase noise characterization of microwave oscillators, frequency discriminator method, Product Note 11729 C-2, Hewlett-Packard Co., P.O. Box 10301, Palo Alto, CA 94303.

[15] "Spectrum analysis . . Noise measurements," Application Note 150 4, Hewlett-Packard Co., P.O. Box 10301, Palo Alto, CA 94303.

[16] W. P. Robins, Phase Noise in Signal Sources, IEE Telecommun. Ser. 9. London: Peter Peregrinus, 1982.

[17] B. Van der Pol, "The nonlinear theory of electric oscillators," Proc. IRE, vol. 22, pp. 1051-1086, Sept. 1934.

[18] R. Alder, "A study of locking phenomena in oscillators," Proc. IEEE, vol. 61, pp. 1380-1385, Oct. 1973.

[19] K. Kurokawa, "Injection locking of microwave solid-state oscillators," Proc. IEEE, vol. 61, pp. 1386-1410, Oct. 1973.

[20] C. J. Buczek, R. J. Freidberg, and M. L. Skolnick, "Laser injection locking," Proc. IEEE, vol. 61, pp. 1411-1431, Oct. 1973.

[21] M. J. W. Rodwell, K. J. Weingarten, D. M. Bloom, T. Baer, and B. H. Kolner, "Reduction of timing fluctuations in a mode-locked Nd: YAG laser by electronic feedback," Opt. Lett., vol. 11, pp. 638640 , Oct. 1986.

[22] D. Cotter, "Technique for highly stable active mode-locking," in Ultrafast Phenomena IV, D. A. Auston and K. B. Eisenthal, Eds. New York: Springer-Verlag, 1984, pp. 78-80.

[23] D. R. Jordan and P. L. Penny, Eds., Transmission Systems for Communications, 5th ed. Bell Lab., 1982. See especially ch. 17 and 24

[24] S. R. Kurtz, "Mixers as phase detectors," Watkins-Johnson Co. Tech Notes, vol. 5, Jan./Feb. 1978, Watkins-Johnson Co., Palo Alto, CA.
[25] D. D. Weiner and J. F. Spina, Sinusoidal Analysis and Modeling of Weakly Nonlinear Circuits. New York: Van Nostrand Reinhold, 1980.

[26] W. J. Rugh, Nonlinear System Theory; The Volterra/Weiner Approach. Baltimore, MD: Johns Hopkins Univ. Press, 1981.

[27] A. van der Ziel, Noise in Solid State Devices and Circuits. New York: Wiley-Interscience, 1986.

[28] Data on high-stability crystal oscillators are available from a number of manufacturers. One supplier is Vectron Laboratories, Inc., 166 Glover Ave., Norwalk, CT 06850.

[29] J. J. D'Azzo and C. H. Houpis, Linear Control System Analysis and Design: Conventional and Modern. New York: McGraw-Hill, 1975.

[30] J. D. Kafka, B. H. Kolner, T. Baer, and D. M. Bloom, "Compression of pulses from a continuous-wave mode-locked Nd: YAG laser,' Opt. Lett., vol. 9, pp. 505-506, 1984.

Mark J. W. Rodwell, for a photograph and biography, see p. 288 of the March 1989 issue of this Journal.

David M. Bloom (S'68-M'76-SM'86-F'87), for a photograph and biography, see p. 288 of the March 1989 issue of this JOURNAL.

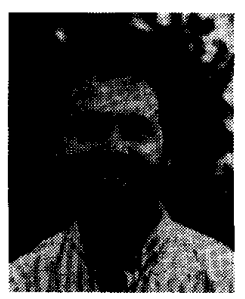

Kurt J. Weingarten (M'88) was born January 30 , 1961 in St. Petersburg, FL. He received the B.S degree from the Georgia Institute of Technology, Atlanta, in 1983 and the M.S. and Ph.D. degrees from Stanford University, Stanford, CA, in 1985 and 1987 , respectively, all in electrical engineering.

While studying for the Ph.D. degree, he worked as a Research Assistant in the Edward L. Ginzton Laboratory, Stanford University. His thesis was entitled, "Gallium arsenide integrated circuit testing using electrooptic sampling." He has authored more than a dozen papers on this topic and coauthored several papers on related topics such as the timing stabilization of mode-locked lasers. In 1985 he received an IBM Predoctoral Fellowship and in 1986 and 1987 the Newport Research Award. He currently works for Lightwave Electronics in Mountain View, CA, developing a commercial version of an optical tester for GaAs IC's. His research interests are electrooptic sampling of GaAs IC's, highspeed electronic testing, and ultrafast optical pulse generation.

Dr. Weingarten is a member of the Optical Society of America. 